PROCEEDINGS OF THE

AMERICAN MATHEMATICAL SOCIETY

Volume 128, Number 9, Pages 2763-2775

S 0002-9939(99)05311-3

Article electronically published on December 7, 1999

\title{
SPLITTING THE CURVATURE OF THE DETERMINANT LINE BUNDLE
}

\author{
SIMON SCOTT \\ (Communicated by Peter Li) \\ Dedicado a la memoria de Hugo Rojas 1973-1997
}

\begin{abstract}
It is shown that the determinant line bundle associated to a family of Dirac operators over a closed partitioned manifold $M=X^{0} \cup X^{1}$ has a canonical Hermitian metric with compatible connection whose curvature satisfies an additivity formula with contributions from the families of Dirac operators over the two halves.
\end{abstract}

\section{INTRODUCTION}

Let $\mathcal{D}$ be a family of Dirac operators over a closed even-dimensional manifold $M$ parameterized by a smooth manifold $B$. Let $M$ be partitioned into codimension 0 submanifolds $M=X^{0} \cup X^{1}$ and let $\mathcal{D}^{0}, \mathcal{D}^{1}$ be the families obtained by restricting $\mathcal{D}$ to the two halves. The purpose of this note is to communicate an additivity formula for the curvature of the determinant line bundle $\mathcal{L}$ associated to $\mathcal{D}$ relative to the partition of $M$ and a choice of elliptic boundary condition $P$ for the families $\mathcal{D}^{0}, \mathcal{D}^{1}$. To do this, we construct a Hermitian metric and compatible connection on $\mathcal{L}$ using a regularized trace and determinant. Full details of the proofs will appear in 12 .

The proof relies on a canonical splitting isomorphism defined for each $P$ between $\mathcal{L}$ and the determinant line bundles associated to the families $\mathcal{D}^{0}$ and $\mathcal{D}^{1}$. A similar but less general splitting isomorphism has been proved by Piazza [7] which leads in the limit to an additivity formula for the $\zeta$-function curvature as the metric is blown-up in a normal direction to $X^{0} \cap X^{1}$. The additivity theorem we prove here requires no asymptotic arguments. This is because the connection we use is constructed directly from boundary data arising from the partition; a different partition defines a different connection. These results are concordant with principles suggested by Topological QFT, and provide an explicit representative for the local anomaly obeying the same splitting principle as the Chern class of the determinant bundle.

For the relation between the metric and connection on $\mathcal{L}$ constructed here to the $\zeta$-function metric and connection [2] in the case of a family of elliptic boundary

Received by the editors September 30, 1998.

1991 Mathematics Subject Classification. Primary 58G20, 58G26; Secondary 81T50.

Key words and phrases. Determinant line bundle, elliptic family, Grassmann section, regularized determinant, splitting principle. 
value problems see [5]. For the case of a family of elliptic boundary value problems in one dimension see [14, 4].

\section{Grassmann Sections and the Determinant Bundle}

Let $\pi: Z \longrightarrow B$ be a smooth fibration of manifolds with fibre diffeomorphic to a closed connected even-dimensional manifold $M$. The tangent bundle along the fibres $T(Z / B)$ is taken to be oriented, spin and endowed with a Riemannian metric $g_{T(Z / B)}$. Let $\mathcal{S}(Z / B)$ be the vertical spinor bundle and $E$ a Hermitian coefficient bundle. Associated to this data one has a smooth elliptic family of Dirac operators $\mathcal{D}=\left\{D_{b}: b \in B\right\}: \mathcal{H} \longrightarrow \mathcal{H}$, where $\mathcal{H}=\pi_{*}(\mathcal{S}(Z / B) \otimes E)$ is the infinite-dimensional Hermitian vector bundle on $B$ whose fibre at $b$ is the Fréchet space of smooth sections $\mathcal{H}_{b}=C^{\infty}\left(M_{b}, \mathcal{S}_{b} \otimes E_{b}\right)$. The $Z_{2}$ bundle grading $\mathcal{H}=\mathcal{F}^{+} \oplus \mathcal{F}^{-}$into positive and negative chirality fields defines families of chiral Dirac operators $\mathcal{D}^{ \pm}: \mathcal{F}^{ \pm} \longrightarrow \mathcal{F}^{\mp}$ and hence two families of finite-dimensional vector spaces $\operatorname{ker}\left(\mathcal{D}^{ \pm}\right)=\left\{\operatorname{ker}\left(D_{b}^{ \pm}\right): b \in B\right\}$. The Quillen determinant line bundle Det Ind $\mathcal{D}^{+}$is a complex line bundle over $B$ with fibre at $b$ canonically isomorphic to the complex line $\wedge^{\max }\left(\operatorname{Ker} \mathcal{D}_{b}^{+}\right)^{-1} \otimes \wedge^{\max } \operatorname{Ker}\left(\mathcal{D}_{b}^{-}\right)($see [2, 9] $)$.

A connection on $\mathcal{H}_{Y}$ is defined as follows [1, 2]. A connection on the fibration means a splitting

$$
T Z=T(Z / B) \oplus T^{H} Z
$$

and specifies an isomorphism $T^{H} Z \cong \pi^{*} T B$. A choice of metric $g_{T B}$ on $T B$ determines a metric $g_{T Z}=g_{T(Z / B)} \oplus \pi^{*} g_{T B}$ on $T Z$ with Levi-Civita connection $\nabla^{T Z}$. We define a connection on $T(Z / B)$ by

$$
\nabla^{T(Z / B)}=P_{Z / B} \nabla^{T Z} P_{Z / B}+c,
$$

where $P_{Z / B}$ is the orthogonal projection on $T Z$ with range $T(Z / B)$ and $c$ is the 1 -form on $Z$ defined by $\left[\frac{1}{2} i_{\xi} d\left(\operatorname{vol}_{Z / B}\right)\right]_{V}=c(\xi) \operatorname{vol}_{Z / B}$. Here $\operatorname{vol}_{Z / B}$ is the volume form defined by $g_{T(Z / B)}$ regarded as an $n$-form on $Z$ and [.] $]_{V}$ means the vertical component. The connection $\nabla^{T(Z / B)}$ lifts to a connection on the vertical spinor bundle, and endowing $E$ with a connection compatible with its metric we obtain a connection $\nabla^{\mathcal{S}(Z / B)}$ on each of the bundles $\mathcal{S}(Z / B) \otimes E, \mathcal{S}^{ \pm}(Z / B) \otimes E$. Because a section of the infinite-dimensional bundle $\mathcal{H}$ is identified as a section of $\mathcal{S}(Z / B) \otimes E$ we have a unitary connection $\nabla^{Z}$ on $\mathcal{H}, \mathcal{F}^{ \pm}$

$$
\nabla_{\xi}^{Z} s=\nabla_{\xi_{H}}^{\mathcal{S}(Z / B)} s,
$$

where $\xi_{H}$ is the horizontal lift of $\xi \in C^{\infty}(B ; T B)$. The correction term $c$ ensures that $\nabla^{Z}$ is unitary.

By a splitting of the fibration $\pi: Z=Z^{0} \cup_{Y} Z^{1} \longrightarrow B$ we mean an embedded connected codimension 1 subfibration of closed manifolds $\partial \pi: Y \longrightarrow B$ defining on each fibre of $Z$ a partition $M_{b}=X_{b}^{0} \cup_{Y_{b}} X_{b}^{1}$. A splitting defines families of chiral Dirac operators $\mathcal{D}^{+}=\mathcal{D}^{0} \cup \mathcal{D}^{1}$ with $\mathcal{D}^{i}=\left\{D_{b}^{i}=D_{b}^{+} \mid X_{b}^{i}: b \in B\right\}$ associated to the fibrations of manifolds with boundary $\pi^{i}: Z^{i} \longrightarrow B$ with $\partial Z^{i}=Y^{i}$. Here the manifolds $Y^{i}$ are identified but such that $Y^{1}$ has the reverse orientation to $Y^{0}=Y$. We assume that $g_{Z / B}$ splits isometrically in a collar neighbourhood $V=[-1,1] \times Y$ of $Y \equiv\{0\} \otimes Y$ as $d u^{2}+g_{Y / B}$, where $u$ is a normal coordinate to $\partial Z$ and $g_{Y / B}$ the induced metric on the boundary fibration, and that the Hermitian metrics on the bundles $S(Z / B), E$ split similarly. In $V_{b}$ the operators $D_{b}^{i}$ have the form $\sigma_{b}\left(\partial / \partial u+A_{b}\right)$ where $\sigma_{b}: S_{Y_{b}} \rightarrow S_{Y_{b}}$ is a unitary bundle isomorphism and $A_{b}$ is an 
elliptic self-adjoint operator identified with the Dirac operator over $Y_{b}$. We make the assumption that the operators of the boundary family

$$
\mathcal{D}_{Y}=\left\{A_{b}: b \in B\right\}: \mathcal{H}_{Y} \longrightarrow \mathcal{H}_{Y}
$$

with $\mathcal{H}_{Y}$ the infinite-dimensional bundle of boundary spinor fields, have kernels of constant rank. (This is convenient, but not essential.) From (1) there is an induced connection on the boundary fibration

$$
T Y=T(Y / B) \oplus T^{H} Y
$$

and hence a connection $\nabla^{Y}$ on $\mathcal{H}_{Y}$ coinciding with the analogue of the construction of $\nabla^{Z}$.

The bundle $\mathcal{H}_{Y}$ is endowed with a $\mathbf{Z}_{2}$-grading $\mathcal{H}_{Y}=\mathcal{H}_{Y}^{+} \oplus \mathcal{H}_{Y}^{-}$defined on each fibre by $\mathcal{H}_{Y, b}=\mathrm{H}_{b}^{+} \oplus \mathrm{H}_{b}^{-}$where $\mathrm{H}_{b}^{+}$(resp. $\mathrm{H}_{b}^{-}$) is the direct sum of the eigenspaces of $A_{b}$ with non-negative (resp. negative) eigenvalues. Associated to the grading we have a (smooth) Grassmann bundle $\mathcal{G} r\left(\mathcal{H}_{Y}\right) \longrightarrow B$ with fibre the infinite-dimensional smooth Grassmannian $\mathcal{G} r_{\infty}\left(\mathrm{H}_{b}\right)$ parameterising projections $P \in \operatorname{End}\left(\mathrm{H}_{b}\right)$ which differ by a smoothing operator from the projection $\Pi_{\geq, b}$ with image $\mathrm{H}_{b}^{+}$, where projection means self-adjoint indempotent. (See [3, 8. 11, 15.) A Grassmann section for the family $\mathcal{D}^{0}$ is defined to be a smooth section $P$ of the fibration $\mathcal{G} r\left(\mathcal{H}_{Y}\right)$. Reversing the orientation on $Y$ defines an opposite Grassmann bundle $\mathcal{G} r\left(\mathcal{H}_{Y^{1}}\right)$, and so, denoting the space of Grassmann sections by $\mathcal{G} r(Y / B)$, we have $P \in \mathcal{G} r(Y / B)$ if and only if $I-P \in \mathcal{G} r\left(Y^{1} / B\right)$. In particular, since $\operatorname{dim} \operatorname{ker} A_{b}$ is assumed to be independent of $b$, the spectral section $\Pi_{\geq}$is an element of $\mathcal{G} r(Y / B)$. Thus any Grassmann section $P \in \mathcal{G} r(Y / B)$ defines an endomorphism of the bundle $\mathcal{H}_{Y}$ which differs from $\Pi_{\geq}$by a smooth family of smoothing operators.

Proposition 2.1. A Grassmann section $P$ is equivalent to a smooth ungraded Fréchet subbundle $\mathcal{W} \longrightarrow B$ of $\mathcal{H}_{Y}$. The bundle $\mathcal{W}$ is endowed with a natural Hermitian metric with compatible connection $\nabla^{\mathcal{W}}=P \cdot \nabla^{Y} \cdot P$ whose curvature is the first-order differential operator

$$
\mathbf{R}^{\mathcal{W}}\left(\xi_{1}, \xi_{2}\right)=P \nabla_{-P_{Y / B}\left[\xi_{1}^{H}, \xi_{2}^{H}\right]}^{\mathcal{S}(Y / B)} \cdot P+P \mathbf{R}^{Y / B}\left(\xi_{1}^{H}, \xi_{2}^{H}\right) P-S_{\mathcal{W}}^{*} \wedge S_{\mathcal{W}},
$$

where $\xi^{H}$ is the horizontal lift of $\xi \in C^{\infty}(B ; T B)$ and $\mathbf{R}^{Y / B}$ is the curvature of $\nabla^{T(Y / B)}$. Here $S_{\mathcal{W}}=\nabla_{\mid \mathcal{W}}^{Y}-\nabla^{\mathcal{W}}$ is the second fundamental form, given locally by $S_{\mathcal{W}}=(I-P)\{d P+\Theta\} P \in \Omega^{1}\left(U, \operatorname{Hom}\left(\mathcal{W}, \mathcal{W}^{\perp}\right)\right.$, where $\Theta$ is the connection 1-form for $\nabla^{Y}$ over $U$.

Proof. $\mathcal{W}$ is the bundle over $B$ with fibre $W_{b}=\operatorname{range}\left(P_{b}\right)$. The first statement follows from the local triviality of $\mathcal{H}_{Y}$ and hence of $\mathcal{G r}\left(\mathcal{H}_{Y}\right)$. The induced metric is defined by $\left\langle s_{1}, s_{2}\right\rangle_{\mathcal{W}}=\left\langle P s_{1}, P s_{2}\right\rangle$, where the right-side is the Hermitian metric on $\mathcal{H}_{Y}$ and it is clear that the connection $\nabla^{\mathcal{W}}$ is compatible. Equation (4) follows easily from [1, Prop. 1.11. The local formula for $S_{\mathcal{W}}$ is an immediate consequence of the definitions.

The curvature of the connection $\nabla^{\mathcal{W}}$ is therefore not of trace-class and so we cannot define a Chern character for $\mathcal{W}$. There is, nevertheless, for each pair of Grassmann sections $P^{0}, P^{1}$ an associated determinant line bundle with well-defined Chern class whose curvature is expressed as the difference of the curvature operators 
of the associated bundles $\mathcal{W}^{0}, \mathcal{W}^{1}$. This is because (seen as operators on $\mathcal{H}_{Y}$ ) they have the same top order symbol, and in fact differ by only a trace-class operator.

To describe these constructions we use a Schatten class calculus, as follows. Let $\operatorname{End}_{c p t}(\mathcal{W})$ denote the algebra of compact bundle endomorphisms (acting on the dense subbundle $\mathcal{W}$ of the $L^{2}$ Hilbert bundle completion $\overline{\mathcal{W}}$ ). The subbundle $\operatorname{End}_{t r}(\mathcal{W})$ is the bundle whose fibre $\operatorname{End}_{t r}\left(W_{b}\right)$ is the ideal (first Schatten class) in $\operatorname{End}_{c p t}\left(W_{b}\right)$ of operators with $\left\|A_{b}\right\|_{t r}=\operatorname{tr}_{W_{b}}\left(A_{b}^{*} A_{b}\right)^{1 / 2}<\infty$, where the trace is the sum over the eigenvalues. Each smooth section of $\operatorname{End}_{t r}(\mathcal{W})$ has an associated trace function $b \mapsto \operatorname{tr}_{W_{b}}\left(A_{b}\right)$. More generally, there is a $\mathbf{Z}$-graded algebra of differential forms taking values in $\operatorname{End}_{t r}(\mathcal{W})$,

$$
\Omega^{k}\left(B, \operatorname{End}_{t r}(\mathcal{W})\right)=\Omega^{k}(B) \otimes \Omega^{0}\left(B, \operatorname{End}_{t r}(\mathcal{W})\right)
$$

with multiplication $\Omega_{t r}^{i} \times \Omega_{t r}^{j} \longrightarrow \Omega_{t r}^{i+j}$ and a smooth linear trace map

$$
\operatorname{Tr}_{\mathcal{W}}: \Omega^{k}\left(B, \operatorname{End}_{t r}(\mathcal{W})\right) \longrightarrow \Omega^{k}(B) \quad \theta_{b} \otimes A_{b} \mapsto \theta_{b} \cdot \operatorname{tr}_{W_{b}}\left(A_{b}\right)
$$

satisfying the supertrace identity

$$
\operatorname{Tr}_{\mathcal{W}}\left(A_{1} A_{2}\right)=(-1)^{\left(\operatorname{deg} A_{1}\right)\left(\operatorname{deg} A_{2}\right)} \operatorname{Tr}_{\mathcal{W}}\left(A_{2} A_{1}\right)
$$

From the pointwise estimate $\left\|\wedge^{r} A_{b}\right\|_{t r} \leq \frac{1}{r !}\left(\left\|A_{b}\right\|\right)^{r}$ we have for each $r \in \mathbf{Z}_{+}$a well-defined algebra map

$$
\Omega^{k}\left(B, \operatorname{End}_{t r}(\mathcal{W})\right) \longrightarrow \Omega^{k}\left(B, \operatorname{End}_{t r}\left(\wedge^{r} \mathcal{W}\right)\right) \quad \theta \cdot A \mapsto \theta \cdot \wedge^{r} A
$$

and a smooth map, the Fredholm determinant,

$$
\Omega^{0}\left(B, \operatorname{End}_{t r}(\mathcal{W})\right) \longrightarrow \Omega^{0}(B), \quad A_{b} \rightarrow \operatorname{det}_{F}\left(1+\epsilon A_{b}\right):=\sum_{r=0}^{\infty} \epsilon^{r} \operatorname{tr}_{\mathcal{W}_{b}}\left(\wedge^{r} A_{b}\right)
$$

The Fredholm determinant is multiplicative on elements in $1+\operatorname{End}_{t r}(\mathcal{W})$, and characterized by the property:

\section{Lemma 2.2.}

$$
\frac{d}{d \epsilon} \operatorname{det}_{F}(1+\epsilon A)_{\mid \epsilon=0}=\operatorname{Tr}_{\mathcal{W}}(A)
$$

There are many different determinant line bundles to compare. First, any $P \in$ $\mathcal{G} r(Y / B)$ defines a smooth family of elliptic boundary value problems

$$
\begin{gathered}
\left(\mathcal{D}^{0}, P\right)=\mathcal{D}^{0}: \operatorname{dom}\left(\mathcal{D}^{0}, P\right) \longrightarrow L^{2}\left(Z^{0}, \mathcal{S}^{-}(Z / B) \otimes E\right), \\
\operatorname{dom}\left(\mathcal{D}^{0}, P\right)=\left\{\psi \in H^{1}\left(Z^{0}, \mathcal{S}^{+}(Z / B) \otimes E\right): P \psi_{\mid Y}=0\right\},
\end{gathered}
$$

where $H^{1}$ is the first Sobolev space. Similarly, we have $\left(\mathcal{D}^{1}, I-P\right)$. The families $\left(\mathcal{D}^{0}, P\right)$ and $\left(\mathcal{D}^{1}, I-P\right)$ define elliptic families of Fredholm operators varying smoothly with the parameters, and we obtain the following fact.

Proposition 2.3. For each $P \in \mathcal{G} r(Y / B)$ there is a Quillen determinant line bundle Det $\operatorname{Ind}\left(\mathcal{D}^{0}, P\right)$ with canonical determinant section $b \mapsto \operatorname{det} D_{P_{b}}^{0}$ non-zero precisely where $D_{P_{b}}^{0}$ is invertible. Similarly, we have $\operatorname{Det} \operatorname{Ind}\left(\mathcal{D}^{1}, I-P\right)$. 
Second, associated to each pair of Grassmann sections $P^{0}, P^{1}$ there is a canonical smooth Fredholm family of generalized Toeplitz operators

$$
\left(P^{0}, P^{1}\right) \in C^{\infty}\left(B ; \operatorname{Hom}\left(\mathcal{W}^{0}, \mathcal{W}^{1}\right)\right), \quad\left(P^{0}, P^{1}\right)_{b} \equiv P_{b}^{1} P_{b}^{0}: W_{0, b} \longrightarrow W_{1, b},
$$

where $\mathcal{W}^{i}$ are the bundles of Proposition 2.1. To define the determinant bundle for such a family needs a more general construction than the Quillen determinant line bundle.

To this end, we adapt a construction of Segal [10]. By an admissible family associated to the fibration $\pi: Z \rightarrow B$ we mean any of the families defined above or associated families or any finite-rank homorphism of vector bundles over $B$. Let $\mathcal{A}=\left\{A_{b}: b \in B\right\}: \mathcal{E}^{+} \longrightarrow \mathcal{E}^{-}$be such a family where $\mathcal{E}^{ \pm}$are the appropriate bundles over $B$. The determinant line $\operatorname{Det}\left(A_{b}\right)$ when ind $A_{b}=0$ is defined to be the set of equivalences classes $[T, \lambda]$ of pairs $(T, \lambda)$ where $T-A_{b} \in \operatorname{Hom}_{t r}\left(\mathcal{E}_{b}^{+}, \mathcal{E}_{b}^{-}\right)$ and $\lambda \in \mathbf{C}$ relative to the equivalence relation $(T q, \lambda) \sim\left(T, \operatorname{det}_{F}(q) \lambda\right)$ for $q \in$ $1+\operatorname{End}_{t r}\left(\mathcal{E}_{b}^{+}\right)$. If ind $A=r$, we define $\operatorname{Det}\left(A_{b}\right)$ to be the determinant line of $A_{b} \oplus 0$ as an operator $\mathcal{E}_{b} \longrightarrow \mathcal{E}^{-} \oplus \mathbf{C}^{r}$ if $r>0$, or $\mathcal{E}_{b} \oplus \mathbf{C}^{-r} \longrightarrow \mathcal{E}^{-}$if $r<0$.

Proposition 2.4. Let $\mathcal{A}$ be an admissible family. Then $\operatorname{DET}(\mathcal{A})=\bigcup_{b \in B} \operatorname{Det} A_{b}$ is a complex line bundle over $B$ with canonical section det non-zero if and only if $A_{b}$ is invertible.

Proof. To prove this we can work over the open covering of $B$ defined by the sets

$$
U_{\alpha}=\left\{b \in B: A_{b}+\alpha(b) \text { invertible }\right\},
$$

where $\alpha$ is a finite-rank section of $\operatorname{Hom}\left(\mathcal{E}^{+}, \mathcal{E}^{-}\right)$. Complex multiplication is defined on $\operatorname{Det}\left(A_{b}\right)$ by $\mu[T, \lambda]=[T, \mu \lambda], \quad \mu \in \mathbf{C}$. For non-zero elements $\left[T_{\alpha(b)}, \lambda\right],\left[T_{\beta(b)}, \lambda\right] \in$ Det $A_{b}$ over $U_{\alpha} \cap U_{\beta}$, we have

$$
\left[T_{\alpha(b)}, \lambda\right]=g_{\alpha \beta}\left(A_{b}\right)\left[T_{\beta(b)}, \lambda\right]
$$

with $g_{\alpha \beta}\left(A_{b}\right)=\operatorname{det}_{F}\left(\left(A_{b}+\alpha(b)\right)\left(A_{b}+\beta(b)\right)^{-1}\right)$. Since $\left(A_{b}+\alpha(b)\right)\left(A_{b}+\beta(b)\right)^{-1}$ $\left.=1+(\alpha(b)-\beta(b))\left(A_{b}+\beta(b)\right)^{-1}\right) \in 1+\operatorname{End}_{t r}\left(\mathcal{W}_{b}\right)$, then from (6) the map $b \mapsto g_{\alpha \beta}\left(A_{b}\right)$ is a well-defined smooth transition function on $U_{\alpha} \cap U_{\beta}$. This defines $\operatorname{DET}(\mathcal{A})$ globally as a complex line bundle over $B$. The global canonical determinant section is det $: B \longrightarrow \operatorname{DET}(\mathcal{A}), \quad b \longrightarrow \operatorname{det} A_{b} \equiv\left[A_{b}, 1\right]$.

Thus, in addition to the determinant bundles $\operatorname{DET}\left(\mathcal{D}^{0}, P\right)$, we now have for each family $\left(P^{0}, P^{1}\right)$ a determinant line bundle $\operatorname{DET}\left(P^{0}, P^{1}\right)$ over $B$. The following technical lemma facilitates subsequent identifications.

Lemma 2.5. Let $\mathcal{A}_{i}=\left\{A_{i, b}: \mathcal{E}_{i, b}^{+} \longrightarrow \mathcal{E}_{i, b}^{-}\right\}$for $i=0,1,2$ be admissible families related by a commutative diagram

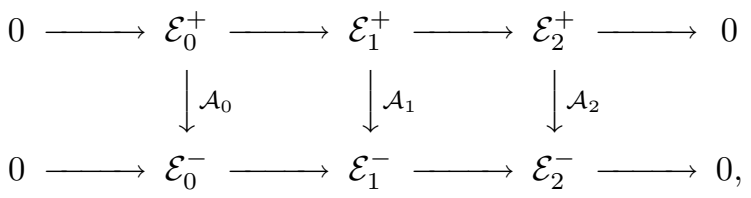

where the rows are short exact sequences of vector bundles, exact on each Sobolev completion. Then there is a canonical isomorphism of determinant line bundles

$$
\operatorname{DET} \mathcal{A}_{1} \cong \operatorname{DET} \mathcal{A}_{0} \otimes \operatorname{DET} \mathcal{A}_{2}
$$

which preserves the determinant sections: $\operatorname{det}\left(A_{1, b}\right) \longleftrightarrow \operatorname{det}\left(A_{0, b}\right) \otimes \operatorname{det}\left(A_{2, b}\right)$. 
In making the identifications of determinant bundles it is convenient to work with the equivalent family of Dirac boundary value problems

$$
\begin{gathered}
\mathcal{D}^{0} \oplus P: H^{1}\left(Z^{0}, \mathcal{S}^{+}(Z / B) \otimes E\right) \longrightarrow L^{2}\left(Z^{0}, \mathcal{S}^{-}(Z / B) \otimes E\right) \oplus \overline{\mathcal{W}} \\
\left(D^{0} \oplus P\right)(\psi)=\left(D^{0} \psi, P\left(\psi_{\mid Y}\right)\right) .
\end{gathered}
$$

The family $\mathcal{D}^{0} \oplus P$ is elliptic with well-defined determinant line bundle $\operatorname{Det} \operatorname{Ind}\left(\mathcal{D}^{0} \oplus P\right)$. Using Lemma 2.5 one can check that it is equivalent to work with either of the constructions of the determinant line bundle for the families $\mathcal{D}^{+},\left(\mathcal{D}^{0}, P\right)$ and $\left(\mathcal{D}^{0} \oplus P\right)$ ( here $\mathcal{D}^{+}$is the family of chiral Dirac operators over the closed manifold $M)$ :

Proposition 2.6. There are canonical isomorphisms of determinant line bundles preserving the determinant sections

$$
\operatorname{Det} \operatorname{Ind} \mathcal{D}^{+} \cong \operatorname{DET} \mathcal{D}^{+},
$$

$$
\operatorname{Det} \operatorname{Ind}\left(\mathcal{D}^{0}, P\right) \cong \operatorname{DET}\left(\mathcal{D}^{0}, P\right) \cong \operatorname{Det} \operatorname{Ind}\left(\mathcal{D}^{0} \oplus P\right) \cong \operatorname{DET}\left(\mathcal{D}^{0} \oplus P\right) .
$$

The following relation is crucial in explaining the sewing properties of determinant line bundles.

Theorem 2.7. Let $P^{0}, P^{1}, P^{2} \in \mathcal{G} r(Y / B)$. Then there is a canonical isomorphism of determinant line bundles

$$
\operatorname{DET}\left(P^{0}, P^{1}\right) \otimes \operatorname{DET}\left(P^{1}, P^{2}\right) \cong \operatorname{DET}\left(P^{0}, P^{2}\right)
$$

$$
\operatorname{det}\left(P^{0}, P^{1}\right) \otimes \operatorname{det}\left(P^{1}, P^{2}\right) \longmapsto \operatorname{det}\left[\left(P^{1}, P^{2}\right) \circ\left(P^{0}, P^{1}\right)\right] .
$$

Proof. Let $\mathcal{W}^{i}, i=0,1,2$, be the bundles defined by the $P^{i}$ and let $U_{\alpha}$ be as in (8). Over $U_{\alpha} \cap U_{\beta} \neq \emptyset$ with $\alpha \in \operatorname{Hom}\left(\mathcal{W}^{0}, \mathcal{W}^{1}\right), \beta \in \operatorname{Hom}\left(\mathcal{W}^{1}, \mathcal{W}^{2}\right)$, the isomorphism is defined by

$$
\operatorname{det}\left(P^{0}, P^{1}\right)_{\alpha} \otimes \operatorname{det}\left(P^{1}, P^{2}\right)_{\beta} \longmapsto \operatorname{det}\left(P^{1}, P^{2}\right)_{\beta} \circ\left(P^{0}, P^{1}\right)_{\alpha} .
$$

Because $\left(P^{1}, P^{2}\right)_{\beta} \circ\left(P^{0}, P^{1}\right)_{\alpha}$ differs from $\left(P^{0}, P^{2}\right)$ by the sum of a family of finiterank operators and a family of smoothing operators, and hence by an family of operators of trace-class, we have $\operatorname{DET}\left(P^{0}, P^{2}\right)=\operatorname{DET}\left[\left(P^{1}, P^{2}\right)_{\beta} \circ\left(P^{0}, P^{1}\right)_{\alpha}\right]$. By an obvious patching argument the proposition is proved.

Alternatively, the result is an immediate corollary of 2.5 applied to the commutative diagram of bundle maps

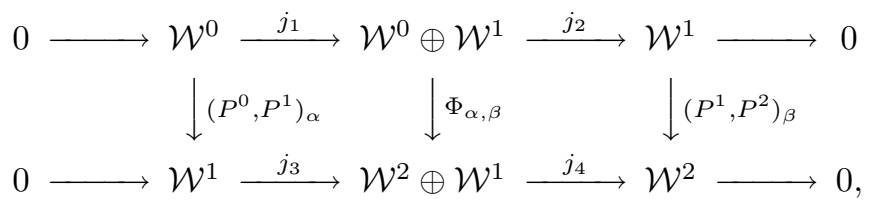

where

$$
\begin{gathered}
P h i_{\alpha, \beta}=\left(P^{1}, P^{2}\right)_{\beta} \circ\left(P^{0}, P^{1}\right)_{\alpha} \oplus I d_{\mathcal{W}^{1}}, \\
j_{1}(\xi)=\left(\xi,\left(P^{0}, P^{1}\right)_{\alpha}(\xi)\right), \\
\left.j_{3}(\xi)=\left(\left(P^{1}, P^{2}\right)_{\beta}(\xi), \xi\right), \quad j_{4}(\xi, \eta)=\xi-\left(P^{0}, P^{1}\right)_{\alpha}(\xi)-\eta, P^{2}\right)_{\beta}(\eta) .
\end{gathered}
$$




\section{SPlitTing ISOMORPHISMS}

There is a canonical smooth Grassmann section which carries global data about the family $\mathcal{D}^{0}$. This is the Calderón section $P\left(\mathcal{D}^{0}\right)$ defined fibrewise by the orthogonal projection $P_{b}\left(\mathcal{D}^{0}\right)$ onto the $L^{2}$ closure of Cauchy data space of harmonic spinors $\mathcal{K}_{b}\left(\mathcal{D}^{0}\right)=\left\{\phi \in \mathcal{H}_{Y, b}: \phi=\psi_{\mid Y_{b}}, \psi \in \mathcal{F}_{b}^{+}, D_{b}^{0} \psi=0\right\}$. That $P\left(\mathcal{D}^{0}\right)$ is an element of $\mathcal{G} r(Y / B)$ depends on the metric $g_{Z / B}$ being a product in a neighbourhood of the boundary $\partial Z$.

Proposition 3.1. Let $P^{0}, P^{1}, P$ be Grassmann sections for the family $\mathcal{D}^{0}$. Then there is a canonical isomorphism of determinant line bundles

$$
\operatorname{Det} \operatorname{Ind}\left(\mathcal{D}^{0}, P^{0}\right) \cong \operatorname{Det} \operatorname{Ind}\left(\mathcal{D}^{0}, P^{1}\right) \otimes \operatorname{DET}\left(P^{1}, P^{0}\right),
$$

which preserves the canonical determinant sections if and only $P^{0}=P^{1}$. One has

$$
\operatorname{Det} \operatorname{Ind}\left(\mathcal{D}^{0}, P\right) \cong \operatorname{DET}\left(P\left(\mathcal{D}^{0}\right), P\right) .
$$

Proof. Let $\mathcal{K}^{0}, \mathcal{W}^{0}$ be the Fréchet bundles over $B$ defined by the Grassmann sections $P\left(\mathcal{D}^{0}\right)$ and $P^{0}$. Then we have the following commutative diagram of bundle maps with exact rows:

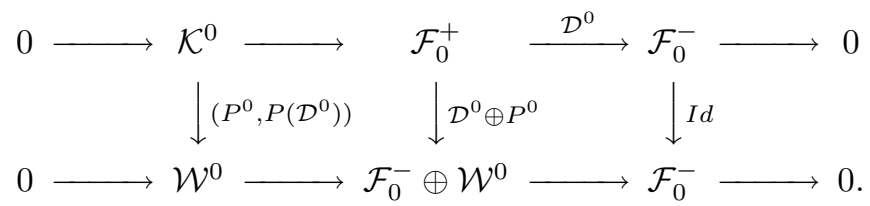

The maps in the lower-row are the obvious ones. To see that the upper-row is exact one needs to know that $\mathcal{D}^{0}$ defines a surjective bundle map and that $\mathcal{K}^{0}$ is canonically identified via the Poisson operator with the bundle with fibre $\operatorname{Ker} D_{b}^{0}$ at $b \in B$. From Lemma 2.5 there is a canonical isomorphism of determinant line bundles

$$
\operatorname{DET}\left(\mathcal{D}^{0} \oplus P^{0}\right) \cong \operatorname{DET}\left(P\left(\mathcal{D}^{0}\right), P^{0}\right) \otimes \operatorname{Det} I d \cong \operatorname{DET}\left(P\left(\mathcal{D}^{0}\right), P^{0}\right) .
$$

Combined with Proposition 2.6 this proves (16), and (15) is now a consequence of Theorem 2.7.

Notice that the analogue of (16) for the family $\mathcal{D}^{1}$ is

$$
\operatorname{Det} \operatorname{Ind}\left(\mathcal{D}^{1}, I-P\right) \cong \operatorname{DET}\left(P, I-P\left(\mathcal{D}^{1}\right)\right) \text {. }
$$

A partition of the closed manifold $M=X^{0} \cup X^{1}$ induces a splitting of the determinant line bundle in the following sense.

Theorem 3.2. For Grassmann sections $P^{0}, P^{1}$ there is a canonical bundle isomorphism

$$
\operatorname{Det} \operatorname{Ind} \mathcal{D}^{+} \cong \operatorname{Det} \operatorname{Ind}\left(\mathcal{D}^{0}, P^{0}\right) \otimes \operatorname{Det} \operatorname{Ind}\left(\mathcal{D}^{1}, I-P^{1}\right) \otimes \operatorname{DET}\left(P^{0}, P^{1}\right) .
$$

The determinant section $\operatorname{det} \mathcal{D}^{+}$of Det Ind $\mathcal{D}^{+}$maps to the determinant section of the bundle on the right-side if and only if $P^{0}=P\left(\mathcal{D}^{0}\right)$ and $P^{1}=I-P\left(\mathcal{D}^{1}\right)$, in which case

$$
\operatorname{Det} \operatorname{Ind} \mathcal{D}^{+} \cong \operatorname{DET}\left(P\left(\mathcal{D}^{0}\right), I-P\left(\mathcal{D}^{1}\right)\right) \text {. }
$$


Note that the isomorphism (19) is completely intrinsic; no choice of a Grassmann section is required. For the isomorphism (18) it is enough to prove (19), the general formula follows from Proposition 3.1 The proof of (19) uses Mayer-Vietoris type arguments; the details will be presented in [12] (see [11] for the single operator case and 10 for the case of $\bar{\partial}$-operators over a Riemann surface).

As a corollary we obtain the following additivity formulae for the Chern class of the determinant line bundle.

Corollary 3.3. In $H^{2}(B)$

$$
\begin{aligned}
& c_{1}\left(\operatorname{Det} \operatorname{Ind}\left(\mathcal{D}^{0}, P^{0}\right)\right)=c_{1}\left(\operatorname{Det} \operatorname{Ind}\left(\mathcal{D}^{0}, P^{1}\right)\right)+c_{1}\left(\operatorname{DET}\left(P^{0}, P^{1}\right)\right), \\
& c_{1}\left(\operatorname{Det} \operatorname{Ind} \mathcal{D}^{+}\right)= \\
& c_{1}\left(\operatorname{Det} \operatorname{Ind}\left(\mathcal{D}^{0}, P^{0}\right)\right)+c_{1}\left(\operatorname{Det} \operatorname{Ind}\left(\mathcal{D}^{1}, I-P^{1}\right)\right) \\
& +c_{1}\left(\operatorname{DET}\left(P^{0}, P^{1}\right)\right) .
\end{aligned}
$$

\section{The CANONiCAL METRIC AND CONNECTION}

We can do better however, and give a local version of the formulas in Corollary 3.3. This requires the construction of a Hermitian metric and compatible connection on the respective determinant bundles using a regularized determinant and trace. One way to do that is to use the $\zeta$-function regularization (see Quillen, Bismut and Freed [9, 2] for closed manifolds, and [7] for $b$-determinant bundles) which by recent results of Grubb [5] and Wojciechowski [16] extends to $\operatorname{Det} \operatorname{Ind}\left(\mathcal{D}^{0}, P\right)$. Here, however, we use a canonical regularization scheme associated to the splitting of the fibration and the Schatten class calculus.

For Grassmann sections $P, P^{0}, P^{1}$ we have the families of Laplacian operators

$$
\Delta^{+}=\left(\mathcal{D}^{+}\right)^{*} \mathcal{D}^{+}, \quad\left(\Delta^{0}, P\right)=\left(\mathcal{D}^{0}, P\right)^{*}\left(\mathcal{D}^{0}, P\right), \quad \Delta_{\left(P^{0}, P^{1}\right)}=\left(P^{0}, P^{1}\right)^{*}\left(P^{0}, P^{1}\right) .
$$

Proposition 4.1. Let $\mathcal{W}^{0}$ be the bundle over $B$ defined by $P^{0}$. Then

$$
\Delta_{\left(P^{0}, P^{1}\right)}-I d \in \Omega^{0}\left(B, \operatorname{End}_{t r}\left(\mathcal{W}^{0}\right)\right) .
$$

There are canonical identifications of determinant bundles

$$
\begin{aligned}
& \operatorname{Det} \operatorname{Ind}\left(\Delta^{0}, P\right) \cong \operatorname{Det} \operatorname{Ind} \Delta_{\left(P\left(D^{0}\right), P\right)}, \\
& \operatorname{Det} \operatorname{Ind} \Delta^{+} \cong \operatorname{Det} \operatorname{Ind} \Delta_{\left(P\left(D^{0}\right), I-P\left(D^{1}\right)\right)}
\end{aligned}
$$

with

$$
\operatorname{det}\left(\Delta^{0}, P\right) \leftrightarrow \operatorname{det} \Delta_{\left(P\left(D^{0}\right), P\right)}, \quad \operatorname{det} \Delta^{+} \leftrightarrow \operatorname{det} \Delta_{\left(P\left(D^{0}\right), I-P\left(D^{1}\right)\right)} .
$$

Proof. Any two Grassmann sections differ by a smooth family of smoothing operators and so the first assertion is obvious.

We prove (23); the proof for (24) is similar. Theorem 2.7 says that associated to the Grassmann sections $P\left(D^{0}\right), P$ there is a canonical isomorphism

$$
\begin{gathered}
\operatorname{DET}\left(P\left(D^{0}\right), P\right) \otimes \operatorname{DET}\left(P, P\left(D^{0}\right)\right) \longrightarrow \operatorname{DET} \Delta_{\left(P\left(D^{0}\right), P\right)} \\
\operatorname{det}\left(P\left(D^{0}\right), P\right) \otimes \operatorname{det}\left(P, P\left(D^{0}\right) \longmapsto \operatorname{det}\left[\left(P, P\left(D^{0}\right)\left(P\left(D^{0}\right), P\right)\right]=\operatorname{det} \Delta_{\left(P\left(D^{0}\right), P\right)} .\right.\right.
\end{gathered}
$$

From (16) there is a canonical isomorphism $\operatorname{DET}\left(P\left(D^{0}\right), P\right) \cong \operatorname{Det} \operatorname{Ind}\left(D^{0}, P\right)$ preserving the determinant sections, and similarly, taking adjoint diagrams, we obtain $\operatorname{DET}\left(P, P\left(D^{0}\right)\right) \cong \operatorname{Det} \operatorname{Ind}\left(D^{0}, P\right)^{*}$ with again $\operatorname{det}\left(P, P\left(D^{0}\right)\right) \leftrightarrow \operatorname{det}\left(D^{0}, P\right)^{*}$. 
Since there is a canonical identification

$$
\operatorname{Det} \operatorname{Ind}\left(D^{0}, P\right) \otimes \operatorname{Det} \operatorname{Ind}\left(D^{0}, P\right)^{*} \longrightarrow \operatorname{Det} \operatorname{Ind}\left(\Delta^{0}, P\right)
$$

which takes $\operatorname{det}\left(D^{0}, P\right) \otimes \operatorname{det}\left(D^{0}, P\right)^{*}$ to $\operatorname{det}\left(\Delta^{0}, P\right)$, the proposition is proved.

From (22) and (25) we can define, relative to the splitting of $M$, a canonical regularization of the Laplacian determinants by

$$
\operatorname{det}_{\mathcal{C}}\left(\Delta^{0}, P\right)=\operatorname{det}_{F} \Delta_{\left(P\left(D^{0}\right), P\right)}, \quad \operatorname{det}_{\mathcal{C}} \Delta^{+}=\operatorname{det}_{F} \Delta_{\left(P\left(D^{0}\right), I-P\left(D^{1}\right)\right)} .
$$

Theorem 4.2. To each splitting $Z=Z^{0} \cup_{Y} Z^{1}$ of the fibration there is a canonical Hermitian metric $\|\cdot\|$ on $\operatorname{Det} \operatorname{Ind} \mathcal{D}^{+}\left(\right.$resp. on $\left.\operatorname{Det} \operatorname{Ind}\left(\mathcal{D}^{0}, P\right)\right)$ with

$$
\left\|\operatorname{det} D_{b}^{+}\right\|^{2}=\operatorname{det}_{\mathcal{C}} \Delta_{b}^{+},
$$

if $\mathcal{D}_{b}^{+}$is invertible and zero otherwise (resp.

$$
\left\|\operatorname{det} D_{P_{b}}^{0}\right\|^{2}=\operatorname{det}_{\mathcal{C}}\left(\Delta^{0}, P\right)_{b}
$$

if $\mathcal{D}_{P_{b}}^{0}$ is invertible and zero otherwise).

Proof. The complex line $\operatorname{DET}\left(P^{0}, P^{1}\right)_{b}$ has a Hermitian inner-product defined by

$$
\left\langle\left[T_{b}, \lambda\right],\left[\tilde{T}_{b}, \mu\right]\right\rangle=\bar{\lambda} \mu \operatorname{det}_{F}\left(T_{b}^{*} \tilde{T}_{b}\right) .
$$

It is easy to check that this definition is independent of the choice of representative $\left(T_{b}, \lambda\right)$ and hence that the inner-product is well defined. Using the inner-product (27) a Hermitian metric is defined on $\operatorname{DET}\left(P^{0}, P^{1}\right)$ over $U_{\alpha}$ by $\left\|\operatorname{det}\left(P^{0}, P^{1}\right)_{\alpha}\right\|^{2}$. It is immediate that this is equal to $\operatorname{det}_{\mathcal{C}} \Delta_{\left(P^{0}, P^{1}\right)_{\alpha}}$. That the locally defined metrics patch together to define a $C^{\infty}$ metric on $\operatorname{DET}\left(P^{0}, P^{1}\right)$ follows from the construction of the determinant line and the transition functions $g_{\alpha \beta}$ of Proposition 2.4 The metric on $\operatorname{Det} \operatorname{Ind}\left(\mathcal{D}^{0}, P\right)$ (resp. Det $\left.\operatorname{Ind} \mathcal{D}^{+}\right)$is the induced metric via the identifications of Proposition 3.1 (resp. Theorem 3.2).

Next we construct a connection compatible with this metric using a regularized trace. Let $\nabla^{0}, \nabla^{1}$ be the unitary connections on the bundles $\mathcal{W}^{0}, \mathcal{W}^{1}$ associated to Grassmann sections $P^{0}, P^{1}$, of Proposition 2.1 and let $\nabla^{\text {Hom }}$ be the induced connection on the bundle $\operatorname{Hom}\left(\mathcal{W}^{0}, \mathcal{W}^{1}\right)$. The crucial fact about the connections $\nabla^{0}, \nabla^{1}$ is that though they do not have local trace-class connection forms, the connection they induce on $\operatorname{End}\left(\mathcal{W}^{0}\right)$ does.

Proposition 4.3. Over $U_{\alpha}$ one has

$$
\left(P^{0}, P^{1}\right)_{\alpha}^{-1} \nabla^{\text {Hom }}\left(P^{0}, P^{1}\right)_{\alpha} \in \Omega^{1}\left(U_{\alpha}, \operatorname{End}_{t r}\left(\mathcal{W}^{0}\right)\right) .
$$

The locally defined connections

$$
\nabla^{\alpha} \operatorname{det}\left(P^{0}, P^{1}\right)_{\alpha}=\operatorname{Tr}_{\mathcal{W}^{0}}\left[\left(P^{0}, P^{1}\right)_{\alpha}^{-1} \nabla^{H o m}\left(P^{0}, P^{1}\right)_{\alpha}\right] \operatorname{det}\left(P^{0}, P^{1}\right)_{\alpha}
$$

patch together into a connection $\nabla^{\text {Det }}$ on $\operatorname{DET}\left(P^{0}, P^{1}\right)$ which is unitary for the metric $\|$.$\| .$

Proof. We may assume that $\mathcal{H}_{Y}$ has been trivialized over the open set $U_{\alpha}$, so that $\nabla^{Y}=d+\Theta$ for some 1-form $\Theta \in \Omega^{1}\left(U_{\alpha}, \operatorname{End}\left(\mathcal{H}_{Y}\right)\right)$. We compute

$$
\begin{gathered}
\left(P^{0}, P^{1}\right)_{\alpha}^{-1} \nabla^{H o m}\left(P^{0}, P^{1}\right)_{\alpha} \\
=\left(P^{0}, P^{1}\right)_{\alpha}^{-1}\left\{d\left(P^{0}, P^{1}\right)_{\alpha}+P^{1} \Theta P^{1}\left(P^{0}, P^{1}\right)_{\alpha}-\left(P^{0}, P^{1}\right)_{\alpha} P^{0} \Theta P^{0}\right\} .
\end{gathered}
$$


Since $P^{1}=P^{0}+\mathcal{S}$ for some smooth family of smoothing operators $\mathcal{S}=\left\{S_{b}\right\}$ : $\mathcal{H}_{Y} \longrightarrow \mathcal{H}_{Y}$, the bracketed term is equal to

$$
\begin{gathered}
d\left(P^{0}, P^{1}\right)_{\alpha}+P^{1} \Theta P^{1} P^{0}+P^{1} \Theta P^{1} \alpha-P^{1} P^{0} \Theta P^{1}-\alpha P^{0} \Theta P^{0} \\
=d P^{0}+d P^{0} \mathcal{S}+P^{0} \mathcal{S}+d \alpha+P^{0} \Theta \mathcal{S} P^{0}+\mathcal{S} \Theta P^{0}-\mathcal{S} \Theta \mathcal{S} \\
-\mathcal{S} P^{0} \Theta P^{0}+P^{0} \Theta P^{0} \alpha+P^{0} \Theta \mathcal{S}+\mathcal{S} \Theta P^{0} \alpha+\mathcal{S} \Theta \mathcal{S} \alpha,
\end{gathered}
$$

which is a sum of smoothing and finite-rank bundle operators, while

$$
\left(\left(P^{0}, P^{1}\right)_{\alpha}\right)^{-1}=\left(P^{0}+P^{0} \mathcal{S}+\alpha\right)^{-1}
$$

and $\mathcal{S}$ is smoothing and $\alpha$ finite-rank. Hence (28) is a sum of smoothing and finite-rank families and hence of trace-class.

The second and third statements are consequences of the identities over $U_{\alpha} \cap U_{\beta}$

$$
\begin{gathered}
d \operatorname{det}_{F}\left(\left(P^{0}, P^{1}\right)_{\beta}^{-1}\left(P^{0}, P^{1}\right)_{\alpha}\right)= \\
\left\{\operatorname{Tr}_{\mathcal{W}^{0}}\left(\left(P^{0}, P^{1}\right)_{\alpha}^{-1} \nabla^{H o m}\left(P^{0}, P^{1}\right)_{\alpha}\right)-\operatorname{Tr}_{\mathcal{W}^{0}}\left(\left(P^{0}, P^{1}\right)_{\beta}^{-1} \nabla^{H o m}\left(P^{0}, P^{1}\right)_{\beta}\right)\right\} \\
\times \operatorname{det}_{F}\left(\left(P^{0}, P^{1}\right)_{\beta}^{-1}\left(P^{0}, P^{1}\right)_{\alpha}\right)
\end{gathered}
$$

and

$$
\begin{gathered}
d \operatorname{det}_{F}\left(\left(P^{1}, P^{0}\right)_{\beta}^{*}\left(P^{0}, P^{1}\right)_{\alpha}\right)= \\
\left\{\operatorname{Tr}_{\mathcal{W}^{0}}\left(\left(P^{0}, P^{1}\right)_{\alpha}^{-1} \nabla^{H o m}\left(P^{0}, P^{1}\right)_{\alpha}\right)-\operatorname{Tr}_{\mathcal{W}^{0}}\left(\left(P^{1}, P^{0}\right)_{\beta}^{-1} \nabla^{H o m}\left(P^{1}, P^{0}\right)_{\beta}\right)\right\}
\end{gathered}
$$

$$
\times \operatorname{det}_{F}\left(\left(P^{1}, P^{0}\right)_{\beta}^{*}\left(P^{0}, P^{1}\right)_{\alpha}\right),
$$

which utilize Lemma 2.2 .

Let $\nabla$ be the induced unitary connection on $\operatorname{Hom}\left(\mathcal{F}^{+}, \mathcal{F}^{-}\right)$from $\nabla^{Y}$. We define a connection on Det Ind $\mathcal{D}^{+}$over $U_{\alpha}$ by

$$
\nabla_{\alpha}^{\left(\mathcal{D}^{+}\right)} \operatorname{det} \mathcal{D}_{\alpha}^{+}=\operatorname{Tr}_{\mathcal{C}}\left[\left(\mathcal{D}_{\alpha}^{+}\right)^{-1} \nabla \mathcal{D}_{\alpha}^{+}\right] \operatorname{det} \mathcal{D}_{\alpha}^{+} .
$$

Here $\operatorname{det}\left(\mathcal{D}_{\alpha}^{+}\right)$is the image of $\operatorname{det}\left(P\left(\mathcal{D}^{0}\right), I-P\left(\mathcal{D}^{1}\right)\right)_{\alpha}$ under the canonical isomorphism (19) (so $\operatorname{det} \mathcal{D}_{\alpha=0}^{+}=\operatorname{det} \mathcal{D}^{+}$) and the connection 1-form is the regularized canonical trace defined by

$$
\operatorname{Tr}_{\mathcal{C}}\left[\left(\mathcal{D}_{\alpha}^{+}\right)^{-1} \nabla \mathcal{D}_{\alpha}^{+}\right]:=\operatorname{Tr}_{\mathcal{K}^{0}}\left[\left(P\left(\mathcal{D}^{0}\right), I-P\left(\mathcal{D}^{1}\right)\right)_{\alpha}^{-1} \nabla^{H o m}\left(P\left(\mathcal{D}^{0}\right), I-P\left(\mathcal{D}^{1}\right)_{\alpha}\right)\right]
$$

where $\mathcal{K}^{0}$ is the bundle over $B$ defined by the Calderón section $P\left(\mathcal{D}^{0}\right)$.

Similarly, a connection can be defined on $\operatorname{Det} \operatorname{Ind}\left(\mathcal{D}^{0}, P\right)$ over $U_{\alpha}$ by

$$
\nabla_{\alpha}^{\left(\mathcal{D}^{0}, P\right)} \operatorname{det}\left(D^{0}, P\right)_{\alpha}=\operatorname{Tr}_{\mathcal{C}}\left[\left(\mathcal{D}^{0}, P\right)_{\alpha}^{-1} \nabla\left(\mathcal{D}^{0}, P\right)_{\alpha}\right] \operatorname{det}\left(D^{0}, P\right)_{\alpha}
$$

where $\left.\operatorname{det}\left(\mathcal{D}^{0}, P\right)_{\alpha}\right)$ is the image of $\operatorname{det}\left(P\left(\mathcal{D}^{0}\right), P\right)_{\alpha}$ under the canonical isomorphism (16) (so $\left.\operatorname{det}\left(\mathcal{D}^{0}, P\right)_{\alpha=0}=\operatorname{det}\left(\mathcal{D}^{0}, P\right)\right)$ and the connection 1-form is the regularized (canonical) trace defined by

$$
\operatorname{Tr}_{\mathcal{C}}\left[\left(\mathcal{D}^{0}, P\right)_{\alpha}^{-1} \nabla\left(\mathcal{D}^{0}, P\right)_{\alpha}\right]:=\operatorname{Tr}_{\mathcal{K}^{0}}\left[\left(P\left(\mathcal{D}^{0}\right), P\right)_{\alpha}^{-1} \nabla^{H o m}\left(P\left(\mathcal{D}^{0}\right), P\right)_{\alpha}\right] .
$$

The 1-forms act as endomorphisms of the determinant line bundles Det Ind $\mathcal{D}_{\mid U_{\alpha}}^{+}$ and $\operatorname{Det} \operatorname{Ind}\left(\mathcal{D}^{0}, P\right)_{\mid U_{\alpha}}$ via the identifications of Section 3 . 
We then have:

Theorem 4.4. For each splitting $Z=Z^{0} \cup_{Y} Z^{1}$ of the fibration the connections defined locally over $U_{\alpha}$ by (32) (resp. (33)) patch together into a connection $\nabla^{\left(\mathcal{D}^{+}\right.}$) on Det Ind $\mathcal{D}^{+}\left(\right.$resp. $\nabla^{\left(\mathcal{D}^{0}, P\right)}$ on $\left.\operatorname{Det} \operatorname{Ind}\left(\mathcal{D}^{0}, P\right)\right)$ compatible with the Hermitian metric.

\section{Additivity of the Curvature}

So far, we know that associated to the fibration of closed manifolds $Z \longrightarrow B$ there is the determinant line bundle Det Ind $\mathcal{D}^{+}$and that when endowed with a splitting $Z^{0} \cup_{Y} Z^{1}$ there is a connection $\nabla^{\left(\mathcal{D}^{+}\right)}$canonically defined on Det Ind $\mathcal{D}^{+}$. If we make a choice of Grassmann section $P \in \mathcal{G} r(Y / B)$, then we have determinant line bundles $\operatorname{Det} \operatorname{Ind}\left(\mathcal{D}^{0}, P\right)$ and $\operatorname{Det} \operatorname{Ind}\left(\mathcal{D}^{1}, I-P\right)$ with unitary connections $\nabla^{\left(\mathcal{D}^{0}, P\right)}$ and $\nabla^{\left(\mathcal{D}^{1}, I-P\right)}$.

Theorem 5.1. Let $\mathbf{R}^{\left(\mathcal{D}^{+}\right)}$be the curvature 2-form of the connection $\nabla^{\left(\mathcal{D}^{+}\right)}$, and let $\mathbf{R}^{\left(\mathcal{D}^{0}, P\right)}, \mathbf{R}^{\left(\mathcal{D}^{1}, I-P\right)}$ be the curvatures of the connections $\nabla^{\left(\mathcal{D}^{0}, P\right)}$ and $\nabla^{\left(\mathcal{D}^{1}, I-P\right)}$. Then

$$
\mathbf{R}^{\left(\mathcal{D}^{+}\right)}=\mathbf{R}^{\left(\mathcal{D}^{0}, P\right)}+\mathbf{R}^{\left(\mathcal{D}^{1}, I-P\right)} .
$$

Note that (34) is taking place in $\Omega^{2}(B)$, since the endomorphism bundle of a complex line bundle is canonically trivial.

Proof. From Theorem 3.2 there is an isomorphism

$$
\operatorname{Det} \operatorname{Ind} \mathcal{D}^{+} \stackrel{\pi}{\longrightarrow} \operatorname{Det} \operatorname{Ind}\left(\mathcal{D}^{0}, P\right) \otimes \operatorname{Det} \operatorname{Ind}\left(\mathcal{D}^{1}, I-P\right)
$$

and hence connections $\nabla^{\left(\mathcal{D}^{+}\right)}$and $\nabla^{\pi}=\pi^{*}\left(\nabla^{\left(\mathcal{D}^{0}, P\right)} \otimes 1+1 \otimes \nabla^{\left(\mathcal{D}^{1}, I-P\right)}\right)$ canonically defined on the determinant line bundle Det Ind $\mathcal{D}^{+}$. The proof of the theorem proceeds over the open sets where $\left(\mathcal{D}^{0}, P\right)_{\alpha}$ and $\left(\mathcal{D}^{1}, I-P\right)_{\beta}$ are invertible. For notational brevity we just do the case over the region $U$ where $\alpha, \beta=0$; note that $\mathcal{D}^{+}$is invertible over $U$. The relation between the corresponding connection 1-forms $\omega^{+}=\operatorname{Tr}_{\mathcal{C}}\left(\mathcal{D}^{+}\right)^{-1} \nabla \mathcal{D}^{+}, \quad \omega_{P}^{0}=\operatorname{Tr}_{\mathcal{C}}\left(\mathcal{D}^{0}, P\right)^{-1} \nabla\left(\mathcal{D}^{0}, P\right)$, and $\omega_{I-P}^{1}=$ $\operatorname{Tr}_{\mathcal{C}}\left(\mathcal{D}^{1}, I-P\right)^{-1} \nabla\left(\mathcal{D}^{1}, I-P\right)$ can be computed by evaluating $\nabla^{\left(\mathcal{D}^{+}\right)}$and $\nabla^{\pi}$ on the section $\operatorname{det} \mathcal{D}^{+}$. Using the identifications of Theorem 2.7 and Section 3 we find that

$$
\omega^{+}=\omega_{P}^{0}+\omega_{I-P}^{1}+d \log F,
$$

where $F$ is the function on $U$ defined by

$$
F(b)=\operatorname{det}_{F}\left[\frac{\left(P\left(\mathcal{D}_{b}^{0}\right), I-P\left(\mathcal{D}_{b}^{1}\right)\right)}{\left(P\left(\mathcal{D}_{b}^{0}\right), P_{b}\right)\left(P_{b}, I-P\left(\mathcal{D}_{b}^{1}\right)\right)}\right] .
$$

Since the connection forms differ by only a closed 1-form, the theorem is proved.

The additivity may also be deduced from the following identities expressing the curvature of the determinant line bundles in terms of the curvatures of the bundles defined by the corresponding Grassmann sections. 
Theorem 5.2. Over $U_{\alpha}$ one has:

$$
\begin{gathered}
\mathbf{R}^{\left(\mathcal{D}^{+}\right)}=\operatorname{Tr}_{\mathcal{K}^{0}}\left[\left(P\left(\mathcal{D}^{0}\right), I-P\left(\mathcal{D}^{1}\right)\right)^{-1} \mathbf{R}^{\mathcal{K}^{1}}\left(P\left(\mathcal{D}^{0}\right), I-P\left(\mathcal{D}^{1}\right)\right)-\mathbf{R}^{\mathcal{K}^{0}}\right], \\
\mathbf{R}^{\left(\mathcal{D}^{0}, P\right)}=\operatorname{Tr}_{\mathcal{K}^{0}}\left[\left(P\left(\mathcal{D}^{0}\right), P\right)^{-1} \mathbf{R}^{\mathcal{W}}\left(P\left(\mathcal{D}^{0}\right), P\right)-\mathbf{R}^{\mathcal{K}^{0}}\right], \\
\mathbf{R}^{\left(\mathcal{D}^{1}, I-P\right)}=\operatorname{Tr}_{\mathcal{W}}\left[\left(P, I-P\left(\mathcal{D}^{1}\right)\right)^{-1} \mathbf{R}^{\mathcal{K}^{1}}\left(P, I-P\left(\mathcal{D}^{1}\right)\right)-\mathbf{R}^{\mathcal{W}}\right] .
\end{gathered}
$$

Here $\mathbf{R}^{\mathcal{K}^{0}}, \mathbf{R}^{\mathcal{K}^{1}}, \mathbf{R}^{\mathcal{W}}$ are the curvature forms of Proposition 2.1 for the bundles $\mathcal{K}^{0}, \mathcal{K}^{1}, \mathcal{W}$ defined by the Grassmann sections $P\left(\mathcal{D}^{0}\right), I-P\left(\mathcal{D}^{1}\right), P$.

Theorem 5.2 may be construed as a statement of the local families index theorem, expressing the curvature of the determinant line bundle as the degree 2 component of a local families index density.

Proof. The essential point is that although the curvature form $\mathbf{R}^{\mathcal{W}}$ defined by a Grassmann section $P$ is not of trace-class, the differences on the right-hand side of (36), (37), (38) are of trace-class. The formulas follow by a direct computation using the local connection forms in (32) and (33).

The additivity (34) now follows from the identities

$$
\begin{aligned}
\operatorname{Tr}_{\mathcal{W}^{0}} & {\left[\mathbf{R}^{\mathcal{W}^{0}}-\left(P^{0}, P^{1}\right)^{-1} \mathbf{R}^{\mathcal{W}^{1}}\left(P^{0}, P^{1}\right)\right] } \\
= & \operatorname{Tr}_{\mathcal{W}^{1}}\left[\left(P^{0}, P^{1}\right) \mathbf{R}^{\mathcal{W}^{0}}\left(P^{0}, P^{1}\right)^{-1}-\mathbf{R}^{\mathcal{W}^{1}}\right], \\
\operatorname{Tr}_{\mathcal{W}^{0}} & {\left[\left(P^{0}, P^{2}\right)^{-1} \mathbf{R}^{\mathcal{W}^{2}}\left(P^{0}, P^{2}\right)-\mathbf{R}^{\mathcal{W}^{0}}\right] } \\
& =\operatorname{Tr}_{\mathcal{W}^{0}}\left[\left[\left(P^{1}, P^{2}\right)\left(P^{0}, P^{1}\right)\right]^{-1} \mathbf{R}^{\mathcal{W}^{2}}\left[\left(P^{1}, P^{2}\right)\left(P^{0}, P^{1}\right)\right]-\mathbf{R}^{\mathcal{W}^{0}}\right]
\end{aligned}
$$

where $P^{i} \in \mathcal{G} r(Y / B)$ define bundles $\mathcal{W}^{i}$ with curvature $\mathbf{R}^{\mathcal{W} i}, i=0,1,2$. The first follows by writing the operators with respect to the direct sum bundle $\mathcal{W}^{0} \oplus \mathcal{W}^{1}$ and using (5), and the second, since the terms differ by an identically vanishing trace over a commutator term.

The additivity is particularly simple in the case where the boundary fibration $\partial Z=Y \longrightarrow B$ is trivial and $\nabla^{Y}$ is the trivial connection, since everything in sight is then trace-class. The connection on $\mathcal{W}$ defined by a Grassmann section $P$ is then $\nabla^{\mathcal{W}}=P \cdot d \cdot P$. From (4) or by direct computation, this has trace-class curvature form with $\operatorname{Tr}_{\mathcal{W}}\left[\mathbf{R}^{\mathcal{W}}\right]=\operatorname{Tr}_{\mathcal{W}}(P d P d P)$. Theorem 5.2 then reduces to:

\section{Theorem 5.3.}

$$
\begin{gathered}
\mathbf{R}^{\left(\mathcal{D}^{+}\right)}=\operatorname{Tr}_{\mathcal{K}^{1}}\left[\mathbf{R}^{\mathcal{K}^{1}}\right]-\operatorname{Tr}_{\mathcal{K}^{0}}\left[\mathbf{R}^{\mathcal{K}^{0}}\right], \\
\mathbf{R}^{\left(\mathcal{D}^{0}, P\right)}=\operatorname{Tr}_{\mathcal{W}}\left[\mathbf{R}^{\mathcal{W}}\right]-\operatorname{Tr}_{\mathcal{K}^{0}}\left[\mathbf{R}^{\left.\mathcal{K}^{0}\right]},\right. \\
\mathbf{R}^{\left(\mathcal{D}^{1}, I-P\right)}=\operatorname{Tr}_{\mathcal{K}^{1}}\left[\mathbf{R}^{\mathcal{K}^{1}}\right]-\operatorname{Tr}_{\mathcal{W}}\left[\mathbf{R}^{\mathcal{W}}\right] .
\end{gathered}
$$




\section{REFERENCES}

[1] Bismut, J.M.: 1986, 'The Atiyah-Singer index theorem for families of Dirac operators: Two heat equation proofs', Invent. Math. 83, 91-151. MR 87g:58117

[2] Bismut, J.M., Freed,D.: 1986 'The analysis of elliptic families:(I) Metrics and connections on determinant bundles', Commun. Math. Phys. 106, 159-176. MR 88h:58110a

[3] Booß-Bavnbek, B., and Wojciechowski, K.P.: 1993, Elliptic Boundary Problems for Dirac Operators, Birkhäuser, Boston. MR 94h:58168

[4] Booß-Bavnbek, B., Scott, S. G., and Wojciechowski, K. P.: 1998, 'The $\zeta$-determinant and $\mathcal{C}$-determinant on the Grassmannian in dimension one', Letters in Math. Phys., to appear.

[5] Grubb, G.: 1999, 'Trace expansions for pseudodifferential boundary problems for Dirac-type operators and more general systems', Ark. Mat. 37, 45-86.

[6] Melrose, R.B., Piazza, P.: 1997, 'Families of Dirac operators, boundaries and the $b$-calculus', J. Diff. Geom. 46, 99-167. MR 99a:58144

[7] Piazza, P.: 1996, 'Determinant bundles, manifolds with boundary and surgery', I Comm. Math. Phys. 178, 597-626; 1998, II, Comm. Math. Phys. 193, 105-124. MR 98a:58169

[8] Pressley, A. and Segal, G.B.: 1986, 'Loop Groups', O.U.P. MR 88i:22049

[9] Quillen, D.G.: 1985, 'Determinants of Cauchy-Riemann operators over a Riemann surface', Funk. Anal. i ego Prilozhenya 19, 37-41. MR 86g:32035

[10] Segal, G.B.: 1990, 'The definition of conformal field theory', preprint.

[11] Scott, S.G.: 1995, 'Determinants of Dirac boundary value problems over odd-dimensional manifolds', Commun. Math. Phys. 173, 43-76. MR 96g:58205

[12] Scott, S.G.: 1997, in preparation.

[13] Scott, S.G.: 1999, 'Determinants of higher-order elliptic boundary value problems and the Quillen metric', preprint.

[14] Scott, S.G., and Torres, F.: 1998, 'Elliptic families in dimension one: geometry of the determinant line bundle', preprint.

[15] Scott, S.G., and Wojciechowski, K.P.: 1998, 'The $\zeta$-Determinant and Quillen's determinant on the Grassmannian of elliptic self-adjoint boundary conditions', $C$. $R$. Acad. Sci. Paris, $t$. 328, Serie I, 139-144.

[16] Wojciechowski, K.P.: 1997, 'The $\zeta$-determinant and the additivity of the $\eta$-invariant on the smooth, self-adjoint Grassmannian', Comm. Math. Phys. 201, 423-444.

Department of Mathematics, King's College, Strand, London WC2R 2LS, United KINGDOM

E-mail address: sscott@mth.kcl.ac.uk 